\title{
Quantum Manifestations of Bifurcations of Closed Orbits in the Photoabsorption Spectra of Atoms in Electric Fields
}

J. Gao

John B. Delos

William \& Mary, jbdelos@wm.edu

Follow this and additional works at: https://scholarworks.wm.edu/aspubs

Part of the Physics Commons

\section{Recommended Citation}

Gao, J. and Delos, John B., Quantum Manifestations of Bifurcations of Closed Orbits in the Photoabsorption Spectra of Atoms in Electric Fields (1997). Physical Review A, 56(1), 356-364. https://doi.org/10.1103/PhysRevA.56.356 accepted for inclusion in Arts \& Sciences Articles by an authorized administrator of W\&M ScholarWorks. For more information, please contact scholarworks@wm.edu. 


\title{
Quantum manifestations of bifurcations of closed orbits in the photoabsorption spectra of atoms in electric fields
}

\author{
J. Gao \\ Department of Chemistry and Physics, Kean College, Union, New Jersey 07083 \\ J. B. Delos \\ Department of Physics, College of William and Mary, Williamsburg, Virginia 23187 \\ and JILA, University of Colorado, Boulder, Colorado 80309
}

(Received 3 June 1996; revised manuscript received 31 January 1997)

\begin{abstract}
The methods developed in the preceding paper (paper II) are used to construct a wave function near a bifurcation of classical orbits of an atomic electron in an electric field. A formula for the recurrence strength near the bifurcation is derived and compared with experimental measurements. [S1050-2947(97)03206-X]
\end{abstract}

PACS number(s): $32.80 . \mathrm{Gc}$

\section{INTRODUCTION}

The preceding paper (paper II) showed how to calculate the effects of recurrences near a bifurcation of closed orbits if the electron is moving in parallel electric and magnetic fields, with no Coulomb attraction to a residual ion. Here we consider recurrences associated with orbits of an electron in an atom in an electric field. We no longer have a magnetic field present - the electron is acted upon by the Coulomb attraction to the ionic core and by the electric field. (There is also a short-range core potential, but that need not be considered.)

When trajectories are drawn in semiparabolic coordinates, they have shapes that are similar to those seen in the preceding papers. In particular, we find bifurcations that occur when a cusp passes through the origin (Fig. 1). We expect, therefore, that a similar theory can be used to describe the wave function near these cusps, and the associated recurrence strength near a bifurcation.

There is a complication in this case. Semiparabolic coordinates are very unlike Cartesian or cylindrical coordinates. The axis $u=0$ coincides in real space with the negative $z$ axis, so it is an axis of cylindrical symmetry. This tells us that the cusp is not a two-dimensional structure for which the wave function is a Pearcey function, but is more analogous to a three-dimensional cusp, for which the wave function is a Fresnel-Bessel integral as described in [1]. Furthermore, the axis $v=0$ coincides in real space with the positive $z$ axis, so it is also an axis of cylindrical symmetry. Our cusp is a geometrical object with cylindrical symmetry about two distinct axes. The only such object that can exist in a threedimensional space is a sphere, but our cusp is certainly not spherical. We would need a space of four Cartesian dimensions to properly embed this cusp. This is one manifestation of the deep relationship between a three-dimensional Coulomb system and a four-dimensional harmonic oscillator.

For this paper, we do not need to pursue this relationship any further. Most of what we need for construction of the wave function was given in the preceding paper. We obtained a local wave function for a cusp that is cylindrically symmetric about one axis. We need to rewrite that local wave function in a manner appropriate to the present context, join it to a global wave function (specifically, connect it to an incoming Coulomb wave), and then modify the result to incorporate the second cylindrical symmetry.

The derivation is given in Secs. II and III, and then we compare to experiments in Sec. IV. A brief report of the results was given in [2].

\section{THE RETURNING WAVE FUNCTION NEAR A BIFURCATION}

We follow the preceding paper [1] as closely as possible. Equations of that paper are denoted, for example, (II.3.1).

\section{A. The Schrödinger equation in semiparabolic coordinates}

In scaled semiparabolic coordinates $(v, u, \tau)$

$$
\begin{gathered}
v=\sqrt{r-z} F^{1 / 4}, \\
u=\sqrt{r+z} F^{1 / 4}, \\
\frac{d t}{d \tau}=\left(u^{2}+v^{2}\right) F^{-3 / 4},
\end{gathered}
$$

the Schrödinger equation is (with $\hat{\hbar}=F^{1 / 4}$ )

$$
-\frac{1}{2} \hat{\hbar}^{2}\left[\frac{\partial^{2}}{\partial v^{2}}+\frac{1}{v} \frac{\partial}{\partial v}+\frac{\partial^{2}}{\partial u^{2}}+\frac{1}{u} \frac{\partial}{\partial u}\right] \psi-\varepsilon\left(v^{2}+u^{2}\right) \psi=2 \psi .
$$

If the energy is near zero and $(v, u)$ are both small, everything in the effective potential can be neglected, and the equation becomes

$$
-\frac{1}{2} \hat{\hbar}^{2}\left[\frac{\partial^{2}}{\partial v^{2}}+\frac{1}{v} \frac{\partial}{\partial v}+\frac{\partial^{2}}{\partial u^{2}}+\frac{1}{u} \frac{\partial}{\partial u}\right] \psi=2 \psi .
$$

This separable equation has product solutions

$$
\psi(v, u)=J_{0}\left(p_{v} v / \hat{\hbar}\right) J_{0}\left(p_{u} u / \hat{\hbar}\right),
$$

with the restriction 


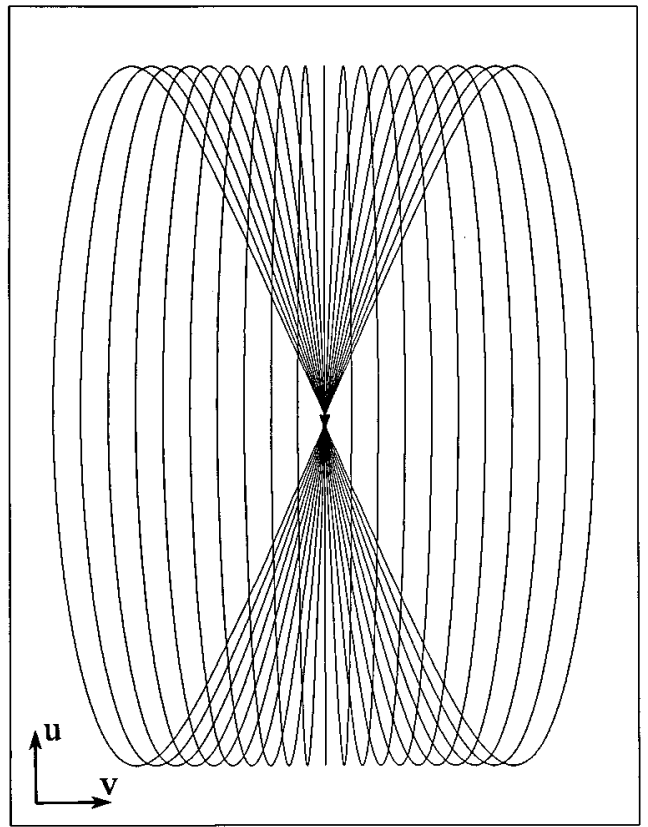

FIG. 1. A family of trajectories going out from the origin at an energy close to the 1:2 bifurcation. One half cycle of $v$ motion (horizontal axis) has a period close to that of one full cycle of $u$ motion (vertical axis). Therefore the trajectories are focused at the origin, forming a small cusp.

$$
p_{v}^{2}+p_{u}^{2}=4 .
$$

Any linear superposition is also a solution, and we can construct any combinations satisfying the restriction $(2.4 \mathrm{~b})$. If we regard $p_{v}$ as the free parameter, and $p_{u}\left(p_{v}\right)$ $=\left(4-p_{v}^{2}\right)^{1 / 2}>0$, then the general superposition of products $(2.4 \mathrm{a})$ is

$$
\psi(v, u)=\int_{0}^{2} \mathcal{H}\left(p_{v}\right) J_{0}\left(p_{v} v / \hat{\hbar}\right) J_{0}\left(p_{u}\left(p_{v}\right) u / \hat{\hbar}\right) d p_{v} .
$$

We need to construct that particular superposition such that the associated rays (trajectories) form a cusp. We will show below that the desired superposition is given by

$$
\begin{aligned}
\mathcal{H}\left(p_{v}\right)= & B \mathcal{Y}_{0}\left(2 \Theta_{p_{0}}\left(p_{v}\right)\right) \mathcal{K}\left(p_{v}\right) \\
& \times \exp \left[\frac{i}{\hat{\hbar}}\left(-\frac{1}{2} a_{1} p_{v}^{2}-\frac{1}{4} a_{3} p_{v}^{4}\right)\right] p_{v}
\end{aligned}
$$

with $B=-\pi 2^{3 / 2} e^{i\left(S_{0} / \hat{\hbar}-\mu_{\|} \pi / 2-\pi\right)} . S_{0}$ is the action of the parallel orbit and $\mu_{\|}$is the Maslov index before the bifurcation of the parallel orbit. The functions $\mathcal{Y}_{0}\left(2 \Theta_{p_{0}}\right)$ and $\mathcal{K}\left(p_{v}\right)$ will be defined later. Physically, they represent the angular distribution of the initial outgoing wave and the semiclassical amplitude of the returning wave in mixed position-momentum space.

The construction closely follows that given in Ref. [1], and proceeds in three steps. (i) If $(v, u)$ were ordinary Cartesian axes, then the cusp would be a two-dimensional object like that described in Sec. III of Ref. [1], and its wave func- tion would be a Pearcey function. (ii) However, looking again at the Schrödinger equation (2.3), the $v$ coordinate is like a cylindrical radius, so the cusp is better regarded as a cylindrically symmetric object, like that described in Sec. IV of paper II. Its wave function should be a Fresnel-Bessel integral analogous to (II.4.13). (iii) Furthermore [again looking at the Schrödinger equation (2.3)], the $u$ coordinate is also like a cylindrical radius, so plane waves like $\exp \left(i p_{z} z / \hat{\hbar}\right) \leftrightarrow \exp \left(i p_{u} u / \hat{\hbar}\right)$ have to be converted to Bessel functions.

\section{B. The wave function for a two-dimensional cusp}

Figures 1 and 2 show the cusp in $(v, u)$ space. If these coordinates $(v, u)$ were an ordinary Cartesian space, then we would have a two-dimensional cusp as described in Sec. III of paper II. All of the formulas therein would hold with the following transcriptions:

$$
\begin{gathered}
x \rightarrow v, \quad z \rightarrow u, \quad p_{x} \rightarrow p_{v}, \quad p_{z} \rightarrow p_{u}, \\
P=\sqrt{2 m E}=\sqrt{p_{u}^{2}+p_{v}^{2}}=2, \\
m=1, \quad t^{\prime} \rightarrow \tau^{\prime}, \quad \theta^{\prime} \rightarrow \Theta^{\prime} .
\end{gathered}
$$

( $\tau^{\prime}$ and $\Theta^{\prime}$ are local parameters of the cusp manifold [1].)

However, certain changes of sign arise because in the present case (for the $\frac{1}{2}$ or $\frac{3}{4}$ bifurcation) $u>0$ as the particle moves through the cusp. Thus Eqs. (II.3.1), (II.3.4a), (II.3.4b), (II.3.5a), and (II.3.7d) become

$$
\begin{gathered}
H\left(p_{v}, p_{u}, v, u\right)=\left(p_{v}^{2}+p_{u}^{2}\right) / 2, \\
\widetilde{S_{2}}\left(p_{v}, u\right)=S_{c}+\left(4-p_{v}^{2}\right)^{1 / 2}\left(u-u_{c}\right)+\frac{1}{4} \alpha p_{v}^{4}, \\
p_{u}\left(p_{v}, u\right)=+\sqrt{4-p_{v}^{2}}, \\
u\left(\tau^{\prime}, \Theta^{\prime}\right)=-u_{c}+2 \tau^{\prime} \cos \Theta^{\prime}+8 \alpha \Theta^{\prime 2}+\ldots .
\end{gathered}
$$

A slightly different representation of $\widetilde{S}$ is more convenient for us,

$$
\widetilde{S_{2}}\left(p_{v}, u\right)=\widetilde{S_{0}}+p_{u}\left(p_{v}\right) u-\frac{1}{2} a_{1} p_{v}^{2}-\frac{1}{4} a_{3} p_{v}^{4} .
$$

$a_{1}$ is related to the tip of the cusp $u_{c}, a_{1}=-u_{c} / p_{u}\left(p_{v}\right)$, and $a_{3}=-\alpha$. See Appendix A for evaluation of these values.

The corresponding configuration-space wave function is

$$
\psi_{2}(v, u)=\frac{1}{\sqrt{-2 \pi i \hat{\hbar}}} \int \exp \left(i p_{v} v / \hat{\hbar}\right) \tilde{\psi}_{2}\left(p_{v}, u\right) d p_{v},
$$

where the semiclassical wave function in the mixed space is

$$
\widetilde{\psi_{2}}\left(p_{v}, u\right)=\widetilde{A_{2}}\left(p_{v}, u\right) \exp \left\{i\left[\widetilde{S_{2}}\left(p_{v}, u\right) / \hat{\hbar}-\nu \pi / 2\right]\right\}
$$

The classical amplitude $\widetilde{A_{2}}\left(p_{v}, u\right)$ is related to the ratio of the Jacobians and initial outgoing wave function $\widetilde{\psi}\left(p_{v_{0}}, u_{0}\right)$ 


$$
\begin{gathered}
\widetilde{A_{2}}\left(p_{v}, u\right)=\widetilde{\psi}\left(p_{v_{0}}, u_{0}\right)\left|\frac{\widetilde{J}_{2}\left(p_{v_{0}}, u_{0}\right)}{\widetilde{J}_{2}\left(p_{v}, u\right)}\right|^{1 / 2}, \\
\widetilde{J}_{2}\left(p_{v}, u\right)=\frac{\partial\left(p_{v}, u\right)}{\partial\left(\tau^{\prime}, \Theta^{\prime}\right)} .
\end{gathered}
$$

$\widetilde{\psi}\left(p_{v_{0}}, u_{0}\right)$ is the initial outgoing wave in the mixed space. Near the cusp, $\psi_{2}(v, u)$ can be reduced to a Pearcey function [Eq. (II.3.21)].

\section{The wave function for a cylindrical cusp}

In this second step we continue to treat $u$ as if it were a Cartesian coordinate (like $z$ ), but we consider the cylindrical nature of the $v$ coordinate. We may imagine sweeping the cusp in Fig. 1 around the $v=0$ axis. Then the associated wave functions are focused onto that axis and are much more intense at $v=0$ than would be predicted by the Pearcey function.

To construct the wave function, we create two fictitious Cartesian $(\bar{x}, \bar{y})$ such that $v=\sqrt{\bar{x}^{2}+\bar{y}^{2}}[3]$. We can then carry over the formulas from Sec. IV of paper II, with the transcriptions

$$
\begin{gathered}
t^{\prime} \rightarrow \tau^{\prime}, \quad \theta^{\prime} \rightarrow \Theta^{\prime}, \quad \varphi^{\prime} \rightarrow \Phi^{\prime}, \\
\rho \rightarrow v, \quad z \rightarrow u, \\
p \rightarrow p_{v}, \quad p_{z} \rightarrow p_{u}, \quad P=\sqrt{p_{u}^{2}+p_{v}^{2}}=2, \\
x \rightarrow \bar{x}, \quad y \rightarrow \bar{y} .
\end{gathered}
$$

Again certain sign changes occur. The local parametric representation of the orbits is similar to Eq. (II.4.2).

$$
\begin{gathered}
\bar{x}\left(\tau^{\prime}, \Theta^{\prime}, \Phi^{\prime}\right)=+2 \tau^{\prime} \sin \Theta^{\prime} \cos \Phi^{\prime}, \\
\bar{y}\left(\tau^{\prime}, \Theta^{\prime}, \Phi^{\prime}\right)=+2 \tau^{\prime} \sin \Theta^{\prime} \sin \Phi^{\prime}, \\
u\left(\tau^{\prime}, \Theta^{\prime}\right)=-u_{c}+2 \tau^{\prime} \cos \Theta^{\prime}+8 \alpha \Theta^{\prime 2}+\cdots,
\end{gathered}
$$

the generator of the Lagrangian manifold is similar to Eqs. (II.4.3c) and (II.4.3d),

$$
\widetilde{S}\left(p_{v}, u\right)=\widetilde{S_{0}}+p_{u}\left(p_{v}\right) u-\frac{1}{2} a_{1} p_{v}^{2}-\frac{1}{4} a_{3} p_{v}^{4},
$$

and the wave function in $(v, u)$ space is similar to Eq. (II.4.13),

$$
\begin{aligned}
\psi(v, u)= & \frac{1}{-i \hat{\hbar}} \int \widetilde{A}\left(p_{v}, u\right) J_{0}\left(p_{v} v / \hat{\hbar}\right) \\
& \times \exp \left(i \left\{\left[\tilde{S}_{0}+p_{u}\left(p_{v}\right) u-\frac{1}{2} a_{1} p_{v}^{2}-\frac{1}{4} a_{3} p_{v}^{4}\right] / \hat{\hbar}\right.\right. \\
& -\nu \pi / 2\}) p_{v} d p_{v} .
\end{aligned}
$$

We have included here the phase associated with the Maslov index in the mixed space, $\nu$.
As always, the amplitude $\widetilde{A}\left(p_{v}, u\right)$ is equal to the wave function on an initial surface times a ratio of Jacobians [Eq. (II.4.10)].

$$
\begin{aligned}
\widetilde{A}\left(p_{v}, u\right) & \equiv \widetilde{A}\left(\overline{p_{x}}, \overline{p_{y}}, u\right) \\
& =\widetilde{\psi}\left(\bar{p}_{x_{0}}, \bar{p}_{y_{0}}, u_{0}\right)\left|\widetilde{J}\left(\bar{p}_{x_{0}}, \bar{p}_{y_{0}}, u_{0}\right) / \widetilde{J}\left(\bar{p}_{x}, \bar{p}_{y}, u\right)\right|^{1 / 2} .
\end{aligned}
$$

The initial points $\left(\bar{p}_{x_{0}}, \bar{p}_{y_{0}}, u_{0}\right)$ are functions of the final points $\left(\bar{p}_{x}, \overline{p_{y}}, u\right)$; each $\left(\bar{p}_{x_{0}}, \bar{p}_{y_{0}}, u_{0}\right)$ is the point on the initial surface from which the trajectories that arrive at $\left(\overline{p_{x}}, \overline{p_{y}}, u\right)$ emanated. The Jacobian is [Eq. (II.4.5a)]

$$
\widetilde{J}\left(\bar{p}_{x}, \bar{p}_{y}, u\right)=\frac{\partial\left(p_{\bar{x}}, p_{\bar{y}}, u\right)}{\partial\left(\tau^{\prime}, \Theta^{\prime}, \Phi^{\prime}\right)}=p_{v} \frac{\partial\left(p_{v}, u\right)}{\partial\left(\tau^{\prime}, \Theta^{\prime}\right)}=p_{v} \widetilde{J}_{2}\left(p_{v}, u\right) .
$$

Just as in the discussion in Sec. V A of paper II, all Jacobians can be expressed in terms of "global" parameters $\left(\tau, \Theta_{0}\right)$ rather than local parameters $\left(\tau^{\prime}, \Theta^{\prime}\right) . \Theta_{0}$ is the initial angle of the outgoing trajectory, $\tau$ is the scaled time with $\tau=0$ at the beginning of the trajectory. Since $\Theta^{\prime}=\Theta_{0}$ and $\tau^{\prime}=\tau$ + const, $\partial\left(\tau^{\prime}, \Theta^{\prime}\right) / \partial\left(\tau, \Theta_{0}\right)=1$, and $\widetilde{J}_{2}\left(p_{v}, u\right)=\partial\left(p_{v}, u\right) /$ $\partial\left(\tau, \Theta_{0}\right)$.

\section{The wave function on the initial surface}

We use the same method as in Sec. V B of paper II, but we get a different result because of the different physical situation. In Ref. [1], as the detached electron moves away from the neutral atom, its wave function is a free-particle outgoing spherical wave (we neglect $F$ and $B$ close to the atom, and there is no long-range Coulomb force). In the present case, the electron occupies a zero-energy outgoing spherical Coulomb wave. (Of course, in both cases there is an angular modulation of the spherical wave arising from the quantum selection rules.)

In configuration space this outgoing wave is [4]

$$
\psi_{\text {out }}\left(r_{0}, \theta_{0}\right)=-i \pi^{1 / 2} 2^{3 / 4} r_{0}^{-3 / 4} e^{-i 3 \pi / 4} e^{i \sqrt{8 r_{0}}} \mathcal{Y}_{0}\left(\theta_{0}\right) \text {. }
$$

Let us reexpress this in terms of the spherical version of scaled semiparabolic coordinates

$$
R=\sqrt{u^{2}+v^{2}}=\sqrt{2 r} F^{1 / 4}, \quad \Theta=\tan ^{-1}(v / u)=\theta / 2 .
$$

Thus

$$
\begin{aligned}
\psi_{\text {out }}\left(r_{0}, \theta_{0}\right) & =\psi_{\text {out }}\left(v_{0}, u_{0}\right) \\
& =-i \pi^{1 / 2} 2^{3 / 2} R_{0}^{-3 / 2} F^{3 / 8} e^{-i 3 \pi / 4} e^{i P R_{0} / \hbar} \mathcal{Y}_{0}\left(2 \Theta_{0}\right)
\end{aligned}
$$

Now thinking of this as a function of $\left(v_{0}, u_{0}\right)$, we transform to momentum space to construct $\widetilde{\psi}_{\text {out }}\left(p_{v_{0}}, u_{0}\right)$. For this purpose we again use the two fictitious Cartesian coordinates and carry out a two-dimensional Fourier transformation by the stationary-phase method, 


$$
\begin{aligned}
\widetilde{\psi}_{\text {out }}\left(p_{v_{0}}, u_{0}\right)= & \widetilde{\psi}_{\text {out }}\left(p_{\bar{x}_{0}}, p_{\bar{y}_{0}}, u_{0}\right) \\
= & \frac{1}{2 \pi i \hat{\hbar}} \int \exp \left[-i\left(p_{\bar{x}} \bar{x}+p_{\bar{y}} \bar{y}\right) / \hat{\hbar}\right] \\
& \times \psi_{\text {out }}\left(\bar{x}, \bar{y}, u_{0}\right) d \bar{x} d \bar{y} \\
= & -i \pi^{1 / 2} 2^{3 / 2} F^{3 / 8} e^{-i 3 \pi / 4} \exp \left[i p_{u_{0}}\left(p_{v_{0}}\right) u_{0} / \hat{\hbar}\right] \\
& \times \mathcal{Y}_{0}\left(2 \Theta_{p_{0}}\right)\left(R_{0}^{1 / 2} P \cos \Theta_{p_{0}}\right)^{-1} .
\end{aligned}
$$

Compared to Eq. (II.5.13), we get different constants and a different angular factor, as would be expected; the more subtle difference is that Eq. (2.22) contains $R_{0}$ $=R_{0}\left(p_{v 0}, u_{0}\right)=u_{0} / \cos \Theta_{p_{0}}$. This is another dependence on the initial boundary which must eventually cancel.

\section{E. The ratio of Jacobians}

As always our Jacobian is initially defined in real Cartesian coordinates and real time $(x, y, z, t)$ [4],

$$
J(x, y, z)=\frac{\partial(x, y, z)}{\partial\left(t, \theta_{0}, \varphi_{0}\right)} .
$$

In terms of the scaled semiparabolic coordinates $(v, u)$ and scaled time $\tau$, it becomes

$$
\begin{aligned}
J(x, y, z) & =J(v, u)=v u \frac{\partial(v, u)}{\partial\left(\tau, \theta_{0}\right)}=\frac{v u}{2} \frac{\partial(v, u)}{\partial\left(\tau, \Theta_{0}\right)} \\
& =\frac{v u}{2} J_{2}(v, u) .
\end{aligned}
$$

The mixed-space Jacobian is obtained by again extending $v \rightarrow(\bar{x}, \bar{y})$,

$$
\widetilde{J}\left(p_{v}, u\right)=J(v, u) \frac{\partial(\bar{x}, \bar{y})}{\partial\left(p_{\bar{x}}, p_{\bar{y}}\right)}=\frac{p_{v} u}{2} \frac{\partial\left(p_{v}, u\right)}{\partial\left(\tau, \Theta_{0}\right)} .
$$

Thus the ratio of Jacobians in Eq. (2.17) becomes

$$
\begin{aligned}
\frac{\widetilde{J}\left(p_{v_{0}}, u_{0}\right)}{\widetilde{J}\left(p_{v}, u\right)} & =\frac{p_{v_{0}} u_{0}}{p_{v} u} \frac{\partial\left(p_{v_{0}}, u_{0}\right)}{\partial\left(\tau, \Theta_{0}\right)} / \frac{\partial\left(p_{v}, u\right)}{\partial\left(\tau, \Theta_{0}\right)} \\
& =\left.\frac{p_{v_{0}} u_{0} p_{u_{0}}}{p_{v} u p_{u}} \frac{\partial p_{v_{0}}}{\partial p_{v}}\right|_{\tau} .
\end{aligned}
$$

Implicit in Eq. (2.26) is the fact that the local parameter $\Theta^{\prime}$, the final angle of each closed orbit, is equal to $\Theta_{0}$, the initial angle for that orbit.

\section{F. The returning wave}

On combining the outgoing wave function with the classical amplitude in the mixed space, we obtain the prefactor of our returning wave function in the mixed space,

$$
\begin{gathered}
\widetilde{A}\left(p_{v}, u\right)=C \mathcal{Y}_{0}\left(2 \Theta_{p_{0}}\right) e^{i p_{u_{0}} u_{0} / \hat{\hbar}} \frac{1}{\sqrt{u p_{u}\left(p_{v}\right)}} \mathcal{K}\left(p_{v}\right), \\
\mathcal{K}\left(p_{v}\right)=\left.\left|\frac{p_{v_{0}}}{p_{v}} \frac{\partial p_{v_{0}}}{\partial p_{v}}\right|\right|_{\tau} ^{1 / 2}, \\
C=-i \pi^{1 / 2} 2 F^{3 / 8} e^{-i 3 \pi / 4},
\end{gathered}
$$

and so, the returning wave function in the mixed space is

$$
\begin{aligned}
\widetilde{\psi}\left(p_{v}, u\right)= & C \mathcal{Y}_{0}\left(2 \Theta_{p_{0}}\right) e^{i p_{u_{0}} u_{0} / \hat{\hbar}} \mathcal{K}\left(p_{v}\right) \frac{e^{-i p_{u}\left(p_{v}\right) u / \hat{\hbar}}}{\sqrt{u p_{u}\left(p_{v}\right)}} \\
& \times \exp \left\{i\left[\left(\widetilde{S_{0}}-\frac{1}{2} a_{1} p_{v}^{2}-\frac{1}{4} a_{3} p_{v}^{4}\right) / \hat{\hbar}-\nu \pi / 2\right]\right\}
\end{aligned}
$$

The $u_{0}$ dependence in the preexponential factor has happily canceled.

As we gaze at Eq. (2.28), we see that the $u$ dependence of $\psi\left(p_{v}, u\right)$ can be correct only for $u$ away from zero. Equation (2.28) is a semiclassical returning wave, and nothing in the argument leading to Eq. (2.28) implies that the formula will be correct everywhere. Indeed we already know from Eq. (2.5) that the correct $u$ dependence must be given by a Bessel function. But Eq. (2.28) contains part of the asymptotic expression for a Bessel function,

$$
\begin{aligned}
J_{0}\left(p_{u} u / \hat{\hbar}\right) \underset{\text { large } u}{\longrightarrow} & \frac{1}{\sqrt{2 \pi p_{u} u / \hat{\hbar}}}\left\{\exp \left[i\left(\frac{p_{u} u}{\hat{\hbar}}-\frac{\pi}{4}\right)\right]\right. \\
& +\exp \left[-i\left(\frac{p_{u} u}{\hat{\hbar}}-\frac{\pi}{4}\right)\right] .
\end{aligned}
$$

Therefore, to make the wave function apply to all values of $u$, we need to replace

$$
\exp \left(-i p_{u} u / \hat{\hbar}\right) / \sqrt{p_{u} u} \rightarrow \sqrt{2 \pi / \hat{\hbar}} e^{-i \pi / 4} J_{0}\left(p_{u} u / \hat{\hbar}\right) .
$$

Thus

$$
\begin{aligned}
\widetilde{\psi}\left(p_{v}, u\right)= & C \mathcal{Y}_{0}\left(2 \Theta_{p_{0}}\right) e^{i p_{u_{0}} u_{0} / \hat{\hbar}} \mathcal{K}\left(p_{v}\right)\left(\frac{2 \pi}{\hat{\hbar}}\right)^{1 / 2} \\
& \times e^{-i \pi / 4} J_{0}\left(\frac{p_{u} u}{\hat{\hbar}}\right) \\
& \times \exp \left\{i\left[\left(\tilde{S}_{0}-\frac{1}{2} a_{1} p_{v}^{2}-\frac{1}{4} a_{3} p_{v}^{4}\right) / \hat{\hbar}-\nu \pi / 2\right]\right\}
\end{aligned}
$$

This wave function now carries the "cylindrical character" of the $u$ coordinate.

To further simplify Eq. (2.31), we note that as in Sec. V C of paper II, the initial and final angles of the trajectories are equal, $\Theta_{0}=\Theta, \Theta_{p_{0}}=\Theta_{p}$ and for $p_{v}=0, S_{0}+p_{u_{0}}\left(p_{v}=0\right) u_{0}$ $=S_{\|}$. The semiclassical theory developed by Maslov tells us 
that when the momentum space index $\nu$ is properly evaluated, all formulas are consistent. We find that our uniform semiclassical results reduce to the earlier primitivesemiclassical formulas if we take $v=\mu_{\|}$(before bifurcation) +2 .

For future reference, we will need to evaluate the preexponential factor at those values of $p_{v}$ that correspond to trajectories that return exactly to the nucleus. One of them is $p_{v}=0$, and at this point

$$
\begin{aligned}
\mathcal{K}\left(p_{v}\right) & =\left.\left|\frac{p_{v_{0}}}{p_{v}} \frac{\partial p_{v_{0}}}{\partial p_{v}}\right|_{\tau}^{1 / 2} \approx\left|\frac{p_{v_{0}}}{p_{v}} \frac{\partial p_{v_{0}}}{\partial p_{v}}\right|_{u_{f}=0}\right|^{1 / 2}=\left|\frac{\partial p_{v_{0}}}{\partial p_{v}}\right|_{u_{f}=0} \\
& =\left|\frac{\partial v}{\partial p_{v}} \frac{\partial p_{v_{0}}}{\partial v}\right|_{u_{f}=0}=\left|\frac{a_{1}}{J_{12}^{0}}\right|
\end{aligned}
$$

where $J_{12}^{0}$, defined in [5], is the element of the monodromy matrix evaluated at the parallel orbit. The other is the newly created closed orbits having $p_{v}= \pm \sqrt{a_{1} / a_{3}}$, and

$$
\begin{aligned}
\mathcal{K}\left(p_{v}\right) & =\left.\left.\left|\frac{p_{v_{0}}}{p_{v}} \frac{\partial p_{v_{0}}}{\partial p_{v}}\right|_{\tau}\right|^{1 / 2} \approx\left|\frac{p_{v_{0}}}{p_{v}} \frac{\partial p_{v_{0}}}{\partial p_{v}}\right|_{u_{f}=0}\right|^{1 / 2} \\
& =\left|\frac{\partial p_{v_{0}}}{\partial p_{v}}\right|_{u_{f}=0}^{1 / 2}=1,
\end{aligned}
$$

as indicated in Ref. [5]. At the bifurcation, $\left|\partial p_{v_{0}} / \partial p_{v}\right|=1$ should apply to both cases. (See Appendix A.)

Finally, the returning wave function in the vicinity of the nucleus (when the cusp is near the nucleus) is obtained by Fourier transformation of $\widetilde{\psi}\left(p_{v}, u\right)$,

$$
\begin{aligned}
\psi(v, u)= & \frac{1}{-2 \pi i \hat{\hbar}} \int \exp \left\{i\left[p_{v} v \cos \left(\varphi-\varphi_{p}\right)\right] / \hat{\hbar}\right\} \\
& \times \widetilde{\psi}\left(p_{v}, u\right) p_{v} d p_{v} d \varphi \\
= & \frac{1}{-i \hat{\hbar}} \int J_{0}\left(p_{v} v / \hat{\hbar}\right) \widetilde{\psi}\left(p_{v}, u\right) p_{v} d p_{v} .
\end{aligned}
$$

More explicitly,

$$
\begin{gathered}
\psi(v, u)=B \int \mathcal{Y}_{0}\left(2 \Theta_{p_{0}}\right) \mathcal{K}\left(p_{v}\right) J_{0}\left(p_{v} v / \hat{\hbar}\right) J_{0}\left(p_{u} u / \hat{\hbar}\right) \\
\times \exp \left[\frac{i}{\hat{\hbar}}\left(-\frac{1}{2} a_{1} p_{v}^{2}-\frac{1}{4} a_{3} p_{v}^{4}\right)\right] p_{v} d p_{v} \\
B=-\pi 2^{3 / 2} e^{i\left(S_{0} / \hat{\hbar}-\mu_{\|} \pi / 2-\pi\right)} .
\end{gathered}
$$

We have thus derived Eqs. (2.5) and (2.6).

\section{OSCILLATOR-STRENGTH DENSITY}

\section{A. Reduction to a Fresnel-type integral}

Now we are ready to derive a refined formula for the oscillator-strength density. The initial wave function is assumed to be

$$
\psi_{i}=R_{n l}(\boldsymbol{r}) Y_{l 0}(\theta, \varphi)
$$

and

$$
D \psi_{i}=r R_{n l}(\boldsymbol{r}) \sum_{l^{\prime}} b_{l^{\prime} 0}^{l} Y_{l^{\prime} 0}(\theta, \varphi)
$$

Later, in comparison with experiments, $3 s$ will be used as the initial state, so

$$
\psi_{i}=R_{30}(\boldsymbol{r}) Y_{00}(\theta, \varphi), \quad D \psi_{i}=\sqrt{1 / 3} r R_{30}(\boldsymbol{r}) Y_{10}(\theta, \varphi)
$$

The oscillator-strength density is proportional to the overlap of the returning wave with the "source" $\left\langle D \psi_{i} \mid \psi\right\rangle$,

$$
D f_{1}=\frac{-2\left(E-E_{i}\right)}{\pi} \operatorname{Im}\left\langle D \psi_{i} \mid \psi\right\rangle,
$$

and

$$
\left\langle D \psi_{i} \mid \psi\right\rangle=2 \pi \int r^{2} d r \int_{0}^{\pi} \sin \theta d \theta\left(D \psi_{i} \psi\right) .
$$

Substituting Eq. (2.35) and changing the order of the integration, we obtain

$$
\begin{aligned}
\left\langle D \psi_{i} \mid \psi\right\rangle= & \int G\left(p_{v}\right)\left\{\int_{0}^{\infty} \int_{0}^{\pi} D \psi_{i} J_{0}\left(p_{u}\left(p_{v}\right) u / \hat{\hbar}\right)\right. \\
& \left.\times J_{0}\left(p_{v} v / \hat{\hbar}\right) r^{2} \sin \theta d r d \theta\right\} p_{v} d p_{v}
\end{aligned}
$$

where

$$
\begin{aligned}
G\left(p_{v}\right)= & 2 \pi B \mathcal{Y}_{0}\left(2 \Theta_{p_{0}}\right) \mathcal{K}\left(p_{v}\right) \\
& \times \exp \left[\frac{i}{\hat{\hbar}}\left(-\frac{1}{2} a_{1} p_{v}^{2}-\frac{1}{4} a_{3} p_{v}^{4}\right)\right] .
\end{aligned}
$$

$\Theta_{p_{0}}$ and $p_{v_{0}}$ are functions of $p_{v}$.

The angular part of the integral is

$$
\begin{aligned}
& A_{\theta}=\int_{0}^{\pi} Y_{l^{\prime} 0}(\theta) J_{0}\left(p_{u} u / \hat{\hbar}\right) J_{0}\left(p_{v} v / \hat{\hbar}\right) \sin \theta d \theta, \\
A_{\theta}= & 4 \int_{0}^{\pi / 2} \sin \Theta \cos \Theta Y_{l^{\prime} 0}(2 \Theta) \\
& \times J_{0}\left(P R \cos \Theta_{P} \cos \Theta / \hat{\hbar}\right) J_{0}\left(P R \sin \Theta_{P} \sin \Theta / \hat{\hbar}\right) d \Theta .
\end{aligned}
$$

In Appendix B we show that this integral is equal to 


$$
A_{\theta}=4(-)^{l^{\prime}} Y_{l^{\prime} 0}\left(\theta_{P}\right) \frac{J_{2 l^{\prime}+1}(\sqrt{8 r})}{(\sqrt{8 r})} .
$$

Then the radial part of the integral

$$
A_{r}=\sum_{l^{\prime}} b_{l^{\prime} 0}^{l} \int_{0}^{\infty} r R_{n l}(r) A_{\theta} r^{2} d r
$$

is proportional to $\mathcal{Y}_{0}\left(2 \Theta_{P}\right)$, since

$$
\begin{aligned}
A_{r} & =\sum_{l^{\prime}} b_{l^{\prime} 0}^{l} 4(-1)^{l^{\prime}} Y_{l^{\prime} 0}(\theta) \int_{0}^{\infty} R_{n l}(r) \frac{J_{2 l^{\prime}+1}(\sqrt{8 r})}{(\sqrt{8 r})} r^{3} d r \\
& =\sqrt{2} \sum_{l^{\prime}}(-1)^{l^{\prime}} I\left(n, l, l^{\prime}\right) b_{l^{\prime} 0}^{l} Y_{l^{\prime} 0}\left(\theta_{P}\right) \\
& =\sqrt{2} \mathcal{Y}_{0}\left(2 \Theta_{P}\right)
\end{aligned}
$$

in which

$$
I\left(n, l, l^{\prime}\right)=\int_{0}^{\infty} R_{n l}(r) \frac{J_{2 l^{\prime}+1}(\sqrt{8 r})}{\sqrt{r}} r^{3} d r .
$$

Now we need to evaluate the integral over $p_{v}$, and thus obtain $\left\langle D \psi_{i} \mid \psi\right\rangle$. On combining Eqs. (3.7) and (3.12), Eq. (3.6) becomes

$$
\begin{aligned}
\left\langle D \psi_{i} \mid \psi\right\rangle= & \int G\left(p_{v}\right) A_{r} p_{v} d p_{v} \\
= & B^{\prime} \int \mathcal{Y}_{0}\left(2 \Theta_{p_{0}}\right) \mathcal{Y}_{0}\left(2 \Theta_{P}\right) \mathcal{K}\left(p_{v}\right) \\
& \times \exp \left[\frac{i}{\hat{\hbar}}\left(-\frac{1}{2} a_{1} p_{v}^{2}-\frac{1}{4} a_{3} p_{v}^{4}\right)\right] p_{v} d p_{v}
\end{aligned}
$$

where

$$
B^{\prime}=2 \pi \sqrt{2} B=-\pi^{2} 2^{3} e^{i\left(S_{0} / \hat{\hbar}-\mu_{\|} \pi / 2-\pi\right)} .
$$

We now have a formula for the oscillator-strength density expressed in terms of an integral of Fresnel type.

\section{B. Uniform asymptotic expansion}

Defining $x=p_{v}^{2}, \lambda=-a_{3} / 2 \hat{\hbar}, a=a_{1} / a_{3}$ we obtain

$$
\left\langle D \psi_{i} \mid \psi\right\rangle=\frac{1}{2} B^{\prime} I(\lambda, a),
$$

where

$$
I(\lambda, a)=\int_{0}^{\infty} g(x) \exp \left[i \lambda\left(\frac{1}{2} x^{2}+a x\right)\right] d x,
$$

with

$$
g\left(p_{v}^{2}\right)=\mathcal{Y}_{0}\left(2 \Theta_{p_{0}}\right) \mathcal{Y}_{0}\left(2 \Theta_{p}\right) \mathcal{K}\left(p_{v}\right)
$$

This is now a Fresnel-type integral, like Eq. (II.4.14). Combining this with Eq. (3.4), we obtain

$$
D f_{1}=\operatorname{Im} B^{\prime \prime} I(\lambda, a)
$$

where

$$
B^{\prime \prime}=\frac{-\left(E-E_{i}\right)}{\pi} B^{\prime}=\left(E-E_{i}\right) \pi 2^{3} e^{i\left(S_{0} / \hat{\hbar}-\mu_{\|} \pi / 2-\pi\right)} .
$$

In some of our calculation we used this formula together with the additional approximation $g\left(p_{v}^{2}\right) \approx g(0)=1$. We also used a slightly more refined version of this approximation: Bleistein [6] wrote down a uniform approximation for integrals like Eq. (3.17).

One can also show that Eq. (3.19) reduces to the usual semiclassical formulas for the parallel orbit and for the new orbit in appropriate limits.

\section{Reduction to semiclassical formulas and consistency check}

As in Sec. V E of paper II, further approximations to the integral formula (3.19) reduce it to previously derived semiclassical formulas. Before the bifurcation, $a_{1}<0$. We use Eqs. (II.4.15c), (2.32), and (A6) to obtain

$$
\begin{aligned}
D f_{1}^{0}= & \left(E-E_{i}\right) 2^{9 / 2} \pi F^{1 / 4} \mathcal{Y}_{0}(0) \mathcal{Y}_{0}(0) \frac{\sqrt{\varepsilon}}{|\sin \sqrt{2 \varepsilon} \tau|} \\
& \times \sin \left(n S_{0} F^{-1 / 4}-\mu_{\|} \pi / 2-\pi / 2\right) .
\end{aligned}
$$

This is exactly the formula for the parallel orbit used in our earlier work.

After the bifurcation, $a_{1}>0$, and the contribution from the parallel orbit arises from the second term Eq. (II. 4.15a). It is identical to Eq. (3.21), except that the Maslov index after the bifurcation has increased by 2 . The contribution of the new orbit arises from the first term in Eq. (II. 4.15a) together with Eqs. (2.33), (A5), and (A7). The result is

$$
\begin{aligned}
D f_{1}^{\text {new }}= & \left(E-E_{i}\right) \pi^{3 / 2} 2^{11 / 2} \mathcal{Y}_{0}\left(\theta_{0}\right) \mathcal{Y}_{0}\left(\theta_{0}\right) F^{1 / 8} \sin \left(\theta_{0} / 2\right) \\
& \times\left(J_{12}^{\text {new }}\right)^{-1 / 2} \sin \left(S^{\text {new }} F^{-1 / 4}-\mu^{\text {new }} \pi / 2\right. \\
& -3 \pi / 2+3 \pi / 4) .
\end{aligned}
$$

This agrees with Eq. (3.3) in Ref. [5].

Finally, at the bifurcation, we use Eq. (II.4.15b) and Ref. [6] to find

$$
\begin{aligned}
D f_{1}= & \left(E-E_{i}\right) \pi^{3 / 2} 2^{3} F^{1 / 8} \mathcal{Y}_{0}(0) \mathcal{Y}_{0}(0)\left(a_{3}\right)^{-1 / 2} \\
& \times \sin \left(S_{0} F^{-1 / 4}-\mu_{\|} \pi / 2-3 \pi / 4\right)+\left(E-E_{i}\right) \pi 2^{4} F^{1 / 4} \\
& \times g^{\prime}(0)\left(a_{3}\right)^{-1} \sin \left(S_{0} F^{-1 / 4}-\mu_{\|} \pi / 2-\pi / 2\right)
\end{aligned}
$$

The first term tells us that the combined recurrence strength of parallel and new orbits at the bifurcation is again half the semiclassical recurrence strength of the new orbit by itself. The second term is a correction arising from Bleistein's improved uniform approximation [6]. In one of our calculations, it turned out to be about $8 \%$ of the primary term. 

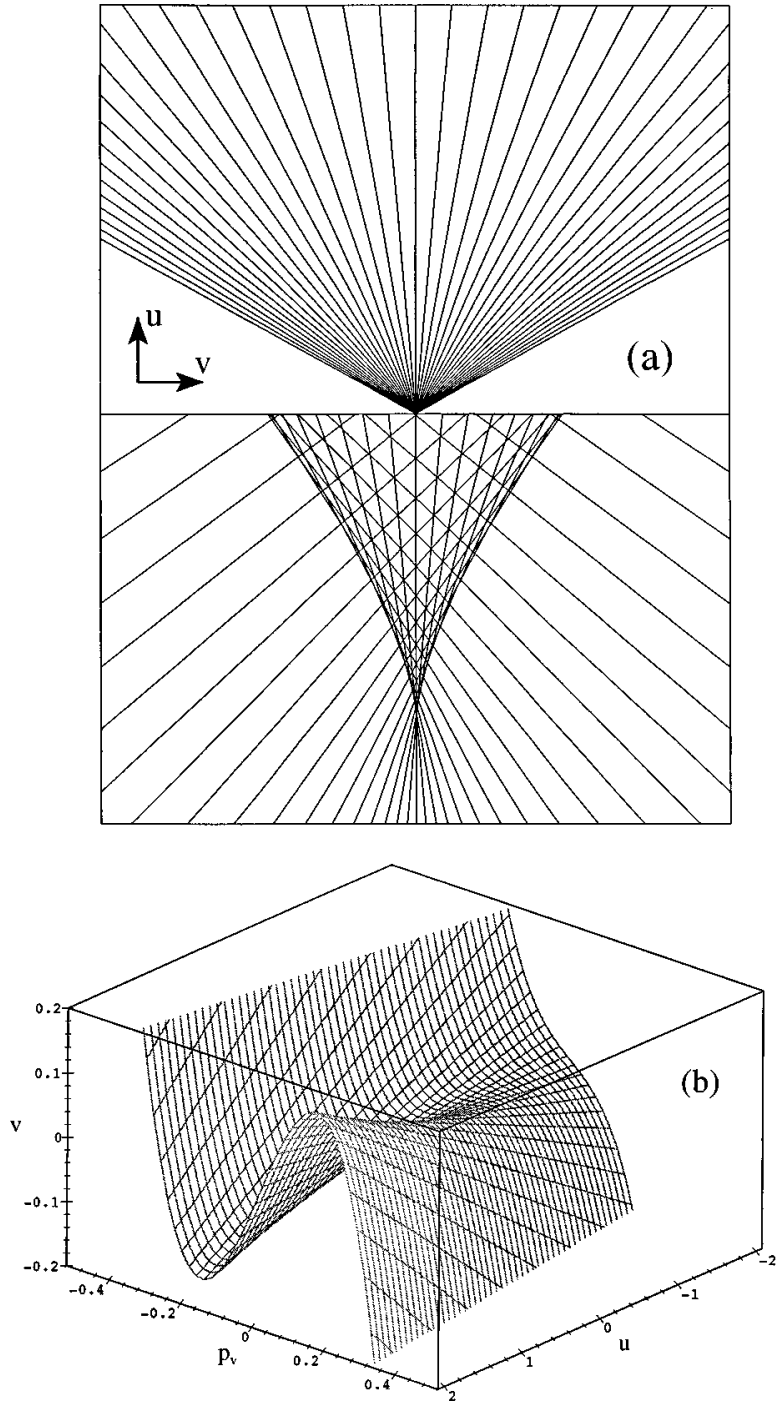

FIG. 2. (a) Enlarged picture of the cusp. Trajectories go out in the upper half of the figure, and return moving upward in the lower half. (b) Lagrangian manifold $v\left(u, p_{v}\right)$ near the cusp.

\section{RECURRENCE SPECTRA AND COMPARISON WITH EXPERIMENTS}

In this section, we compare our calculations with experimental measurements performed by Courtney et al. [2].

The experiment was performed on $m=0$ states of lithium for a number of scaled energies between $\varepsilon=-2.1$ and -0.37 . The experiment employed a lithium atomic beam which passed through holes in the center of a pair of electric plates. Between the field plates, the atoms were excited from the $2 s$ state to the $3 s$ state by a two-photon transition. A second laser was polarized parallel to the applied field and excited the atoms to Rydberg states. Recurrence spectra were obtained by Fourier transforming the measured photoabsorption cross sections.

The initial state is given by Eqs. (3.3). The oscillatorstrength density can be calculated by using the hydrogen model with $3 s$ initial state, since the effect of the phase shift caused by the quantum defect of lithium is small. The reduced recurrence spectrum can then be calculated according to Eqs. (3.11), (4.1), and (4.2) of Ref. [5]. This calculated
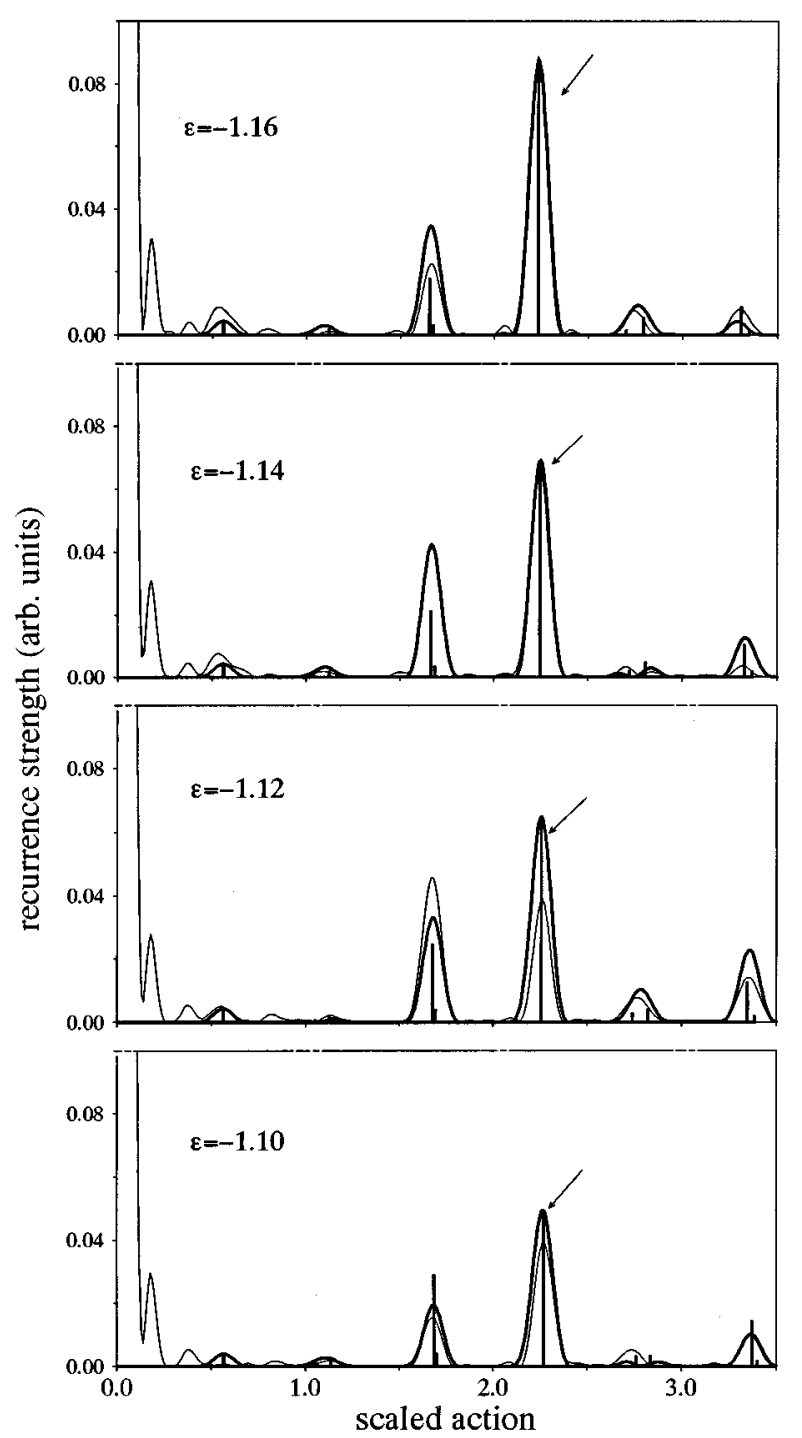

FIG. 3. Recurrence strength near the 3:4 bifurcation. Needles are theory, fine line is experiment. Heavy line is calculated by smoothing the needles consistent with the experimental measurements. One overall multiplicative constant is used to normalize experiments and theory in this figure. All peaks are calculated using the "primitive"'-semiclassical approximation except the one marked with the arrow. That one is the fourth recurrence of the parallel orbit and the new orbit that bifurcates from it. The combined recurrence strength is calculated from Eq. (3.19).

hydrogen spectrum should differ from the lithium spectrum only by a constant.

We show the comparison of calculated recurrence spectra with measurements in small steps of $\varepsilon$ near the $\frac{1}{2}$ and $\frac{3}{4}$ bifurcations. The same normalization constant is used at every energy in each figure (different normalization for the two figures, because the experiments are not absolute). Thick lines are calculations and thin lines are measurements. The needles are the strength of individual orbits and their repetitions.

In Fig. 3, near $\frac{3}{4}$ bifurcation, the uniform approximation is used for the fourth recurrence, and the semiclassical approximation is used for all the other recurrences. Respectable agreement is found between theory and experiment.

In Fig. 4, near $\frac{1}{2}$ bifurcation, the uniform approximation is 


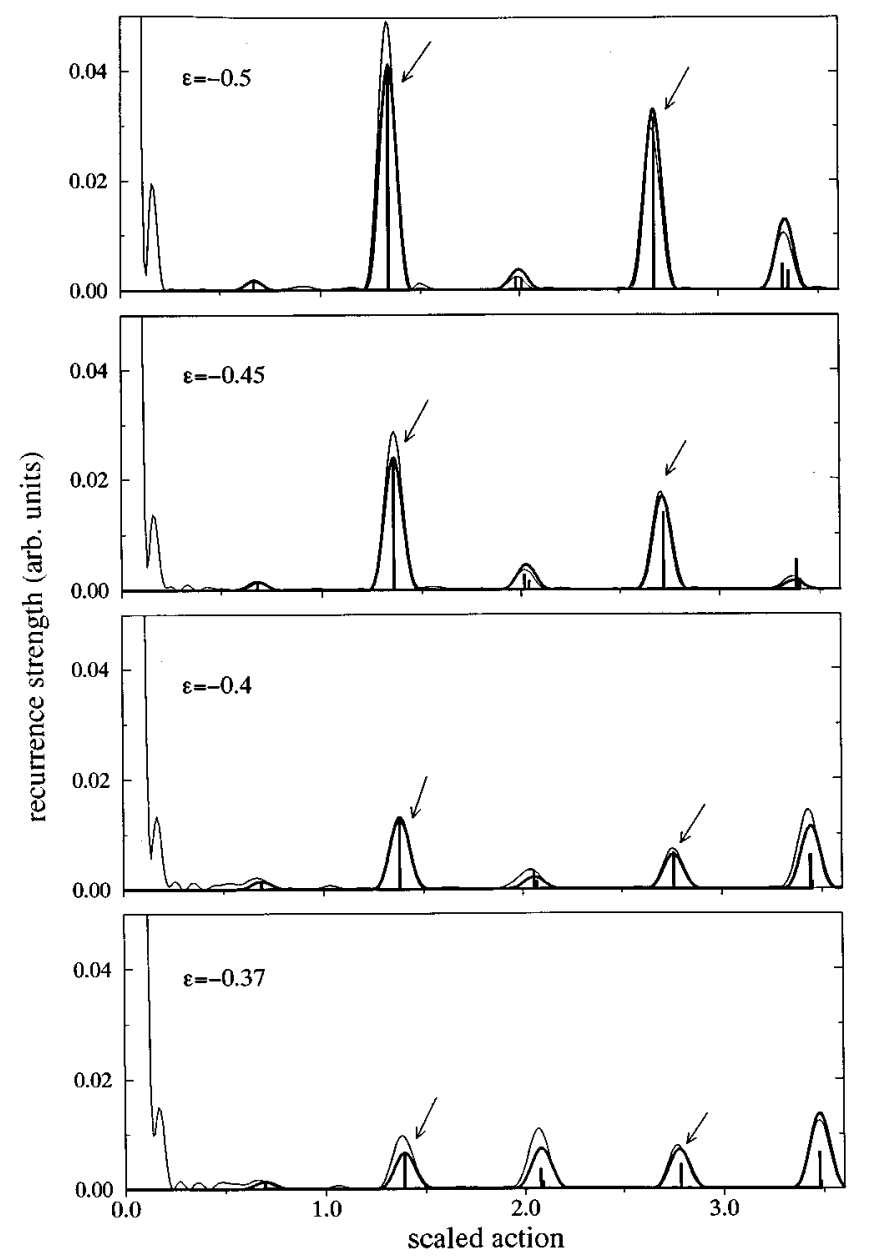

FIG. 4. Same as Fig. 3, near 1:2 bifurcation. The peaks marked with arrows are the second and fourth recurrences of the parallel orbit and the associated bifurcated orbit.

used for the second recurrence and its repetition. Experimental measurements should be most reliable for this case, since they were made repeatedly in small steps specifically for the purpose of studying the bifurcation. Here the agreement between theory and experiment is very good.

As discussed elsewhere, the maximum recurrence strength does not occur right at the bifurcation $(\varepsilon=-1.12$ for the $\frac{3}{4}$ case or $\varepsilon=-0.4$ for the $\frac{1}{2}$ case), but a little after the bifurcation, which agrees with the prediction of Eq. (3.23).

\section{CONCLUSION}

A refined formula for the oscillator-strength density is derived by using a uniform semiclassical approximation. This formula is especially useful near bifurcations where the semiclassical formulas diverge. Comparisons between theory and experiments show very good agreement both near and away from a bifurcation.

\section{ACKNOWLEDGMENTS}

This research was supported by the National Science Foundation and the Office of Naval Research (W\&M) and by Research Corporation and the Petroleum Research Fund (Kean). J.B.D. thanks JILA for their hospitality.

\section{APPENDIX A: EVALUATION OF $a_{1}$ AND $a_{3}$}

From Eq. (9.22b) of Ref. [4] if the initial and final boundary circles are shrunk to zero,

$$
v\left(\tau, \theta_{0}\right)=\sqrt{1-\cos \theta_{0} /|\varepsilon|} \sin (\sqrt{2|\varepsilon|} \tau), \quad \varepsilon<0 .
$$

Thus

$$
p_{v}\left(\tau, \theta_{0}\right)=2 \sin \left(\theta_{0} / 2\right) \cos (\sqrt{2|\varepsilon|} \tau)
$$

To obtain $a_{1}$, we consider the function

$$
\begin{gathered}
v\left(p_{v}, u=0\right)=-\frac{\partial \widetilde{S}\left(p_{v}, u=0\right)}{\partial p_{v}}=a_{1} p_{v}+a_{3} p_{v}^{3}+\cdots, \\
a_{1}=\left.\frac{\partial v}{\partial p_{v}}\right|_{\substack{u=0 \\
p_{v}=0}}=\frac{\left[\partial v\left(\tau, \theta_{0}\right) / \partial \theta_{0}\right]_{\tau}}{\left[\partial p_{v}\left(\tau, \theta_{0}\right) / \partial \theta_{0}\right]_{\tau}}=\frac{\sin (\sqrt{2|\varepsilon|} \tau)}{\sqrt{2|\varepsilon|} \cos (\sqrt{2|\varepsilon|})} .
\end{gathered}
$$

(The derivative at fixed $u$ and the derivative at fixed $\tau$ are equal at $p_{v}=0$.) After bifurcation $a_{3}$ is related to $a_{1}$ through the newly created orbit,

$$
p_{v}=2 \sin \left(\theta_{0} / 2\right) \approx \sqrt{\left|a_{1} / a_{3}\right|} .
$$

Before the bifurcation, however, $a_{3}$ is obtained by extrapolation.

The parameter $a_{1}$ is also related to an element of the monodromy matrix of the parallel orbit. Consider the derivative of $v$ with respect to the initial $p_{v}$ :

$$
\begin{aligned}
J_{12}^{0} & =\left.\frac{\partial v}{\partial p_{v_{0}}}\right|_{\substack{u=0, p_{v}=0}}=\frac{\partial v\left(\tau, \theta_{0}\right) / \partial \theta_{0}}{\partial p_{v_{0}}\left(\tau, \theta_{0}\right) / \partial \theta_{0}}=\frac{\sin (\sqrt{2|\varepsilon|} \tau)}{\sqrt{2|\varepsilon|}} \\
& =\frac{a_{1}}{\cos (\sqrt{2|\varepsilon|} \tau)} .
\end{aligned}
$$

$a_{1}$ is related to the element of the monodromy matrix evaluated at the new orbit, $J_{12}^{\text {new }}$, as well. Whenever Eq. (A3) can be truncated at the cubic level, we find

$$
J_{12}^{\mathrm{new}} \approx \pm 2 a_{1}
$$

Proof:

$$
\pm 1=\left.\frac{\partial p_{v_{0}}}{\partial p_{v}}\right|_{u=0}=\left.\frac{\partial v / \partial p_{v}}{\partial v / \partial p_{v_{0}}}\right|_{u=0} .
$$

Evaluating the derivative of Eq. (A3) at the stationary-phase point $p_{v}^{2} \approx-a_{1} / a_{3}$, we find $\partial v / \partial p_{v}=-2 a_{1}$ and $\partial v / \partial p_{v_{0}}$ is the element of the monodromy matrix $J_{12}^{\text {new }}$. 


\section{APPENDIX B: INTEGRAL INVOLVING BESSEL FUNCTIONS}

In this appendix, we prove the following result:

$$
\begin{aligned}
A_{\theta} / 4= & \int_{0}^{\pi / 2} \sin \Theta \cos \Theta Y_{l^{\prime} 0}(2 \Theta) \\
& \times J_{0}\left(P R \cos \Theta_{P} \cos \Theta / \hat{\hbar}\right) J_{0}\left(P R \sin \Theta_{P} \sin \Theta / \hat{\hbar}\right) d \Theta \\
= & (-1)^{l^{\prime}} Y_{l^{\prime} 0}\left(\theta_{P}\right) \frac{J_{2 l^{\prime}+1}(P R / \hat{\hbar})}{(P R / \hat{\hbar})} \\
= & (-1)^{l^{\prime}} Y_{l^{\prime} 0}\left(\theta_{P}\right) \frac{J_{2 l^{\prime}+1}(\sqrt{8 r})}{\sqrt{8 r}},
\end{aligned}
$$

where $\theta=2 \Theta$ and $\theta_{P}=2 \Theta_{P}$.

Referring to Eq. (3.8), the product $J_{0}\left(p_{u}\left(p_{v}\right) u\right.$ / $\hat{\hbar}) J_{0}\left(p_{v} v / \hat{\hbar}\right)$ is a solution to the zero-energy Schrödinger equation (2.3), and Eq. (3.8) represents the projection of that solution onto the spherical harmonic $Y_{l^{\prime} 0}(\theta)$. The projection is a function of $r$ which must still satisfy the zero-energy Schrödinger equation with angular momentum $l$, i.e., it must be the same constant times $J_{2 l^{\prime}+1}(\sqrt{8 r}) / \sqrt{8 r}$. We evaluate the constant by going to large $r$ and using the stationaryphase approximation.

Using the asymptotic formula for the Bessel function, at large $R$ Eq. (B2) becomes

$$
\begin{aligned}
& (-1)^{l^{\prime}} Y_{l^{\prime} 0}\left(2 \Theta_{P}\right) \frac{J_{2 l^{\prime}+1}(P R / \hat{\hbar})}{(P R / \hat{\hbar})} \\
& =Y_{l^{\prime} 0}\left(2 \Theta_{P}\right)\left(\frac{1}{2 \pi}\right)^{1 / 2}\left(\frac{P R}{\hat{\hbar}}\right)^{-3 / 2}
\end{aligned}
$$

$$
\begin{aligned}
& \times\left\{\exp \left[i\left(\frac{P R}{\hat{\hbar}}-\frac{3}{4} \pi\right)\right]\right. \\
& \left.+\exp \left[-i\left(\frac{P R}{\hat{\hbar}}-\frac{3}{4} \pi\right)\right]\right\} .
\end{aligned}
$$

Likewise, the two Bessel functions become

$$
\begin{aligned}
& J_{0}\left(P R \cos \Theta_{P} \cos \Theta / \hat{\hbar}\right) J_{0}\left(P R \sin \Theta_{P} \sin \Theta / \hat{\hbar}\right) \\
& =\frac{1}{2 \pi P R / \hat{\hbar}} \sqrt{\frac{1}{\cos \Theta_{P} \cos \Theta \sin \Theta_{P} \sin \Theta}} \\
& \quad \times\left\{\exp \left[i\left(\frac{P R}{\hat{\hbar}} \cos \left(\Theta_{P}-\Theta\right)-\frac{\pi}{2}\right)\right]\right. \\
& +\exp \left[-i\left(\frac{P R}{\hat{\hbar}} \cos \left(\Theta_{P}-\Theta\right)-\frac{\pi}{2}\right)\right] \\
& \quad+\exp \left[i \frac{P R}{\hat{\hbar}} \cos \left(\Theta_{P}+\Theta\right)\right] \\
& \left.\quad+\exp \left[-i \frac{P R}{\hbar} \cos \left(\Theta_{P}+\Theta\right)\right]\right\} .
\end{aligned}
$$

On plugging in Eq. (B4), Eq. (B1) is now separated into four integrals with two stationary-phase points $\Theta=\Theta_{P}$ and $\Theta=\pi+\Theta_{P}$ for each of the first two terms, and two more $\Theta=-\Theta_{P}$ and $\Theta=\pi-\Theta_{P}$ for last two terms. However, only $\Theta=\Theta_{P}$ contributes, since the value of both $\Theta$ and $\Theta_{P}$ is in the range of $(0, \pi / 2)$. Using the stationary-phase approximation, the sum of the first two terms reduces to Eq. (B3).
[1] A. D. Peters, C. Jaffé, J. Gao,and J. B. Delos, preceding paper, Phys. Rev. A 56, 345 (1997).

[2] M. Courtney, H. Jiao, N. Spellmeyer, D. Kleppner, J. Gao,and J. Delos, Phys. Rev. Lett. 74, 1538 (1995).

[3] Note that $(\bar{x}, \bar{y})$ are not two of the original Cartesian coordinates $(x, y)$ for the electron. Instead, $(\bar{x}, \bar{y})$ are created in order to convert the $v$ part of the Schrödinger equation into a Car- tesian form. We are making use of the deep relation between a three-dimensional Coulomb problem and a four-dimensional harmonic oscillator.

[4] J. Gao and J. B. Delos, Phys. Rev. A 46, 1455 (1992).

[5] J. Gao and J. B. Delos, Phys. Rev. A 49, 869 (1994).

[6] N. Bleistein, Commun. Pure Appl. Math. XIX, 353 (1966). 